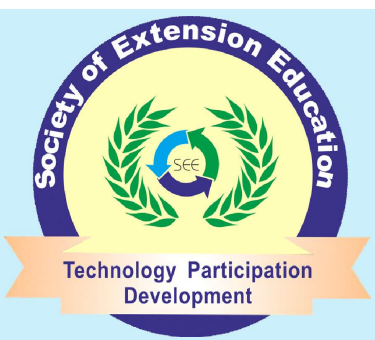

Research Article

\section{Indian Research Journal of Extension Education}

ISSN: 0972-2181 (Print), 0976-1071 (Online)

NAAS Rating : 5.22

Journal homepage: seea.org.in

https://doi.org/10.54986/irjee/2022/jan_mar/92-96

\title{
An Analysis of Social Network Activities of College Students
}

\begin{abstract}
G. Padmini Devi ${ }^{1}$ and Sirisha Deepthi Sornapudi ${ }^{2}$
1. Asstt. Prof. (HSc.), Govt.College for Women (A), Guntur, Andhra Pradesh, 2. Ph.D. Scholar (Textiles and Apparel Designing), CCASc.), MPUAT, Udaipur, Rajasthan.

Corresponding author e-mail : padmini@gcwguntur.ac.in

Paper Received on September 15, 2021, Accepted on Novembe 28, 2021 and Published Online on January 01, 2022

ABSTRACT

The present exploratory study was taken up to know about the various social networking sites that students use and the type of information shared by them on them. WhatsApp emerged as the most preferred medium for sharing messages, photos, and videos. There was a significant difference between the use of social media networks and the content shared on them. Content related to music, cooking, and movies were watched on the top three social networking sites namely YouTube, WhatsApp, and Instagram. The study found a significant difference between watched social media networks. There exists a significant difference regarding the different contents watched on social media. A significant difference between watched and shared content in different social media networks was also established. The three most important advantages of social media as perceived by the students were convenience in keeping in touch with friends, ease to learn new technology, knowledge of various academic institutions for higher studies across the country. Three disadvantages indicated by the study group were less physical activity, cybercrime, and privacy issues.
\end{abstract}

Key words : Social media; College students; Social networking sites.

$\mathbf{I}_{\text {n the present context, ICT plays an influential }}$ role in all walks of life, especially in the education sector (Anil and Godara, 2020). Social media networks are one of the powerful tools of ICT that can be used for multiple purposes. College students are no strangers to technology, the use of which is rampant and varied among the student community. Many technological devices like mobile, laptop, etc., are used both for personal and academic purposes. Smartphones and the internet make it easy for students to connect to family, friends, and colleagues through various social networking sites. The time spent on these social networking sites has an impact on the quality of life of students. It affects their physical, mental, and spiritual health (Saini et al., 2020). Social media transcend the confines of the geographical barriers and help connect people globally. Social network plays a pivotal role in the life of a student by building, maintaining or even reviving relationships and improving students' learning skills (Tayseer et al., 2014). A large portion of users on social networking sites is students. Connecting to their network through social media has emerged as the new normal in the post-Covid era. While there are positive and negative aspects to this form of communication, its impact on young minds cannot be undermined. Consequently, parents, teachers, and educational institutions should try to use it as a principal means of communication, given that many studies 
establish the fact that students spend a major portion of their time on them. Interactions on social media are bound to have a lasting impact on identity, privacy, social capital, youth culture, their emotional well-being, education, and interpersonal relationships (Jha, 2017).

There was no significant difference between daily and non-daily users' physical and social health problems (Saini et al., 2020). The use of social media by the teachers and students and its impact on the teaching learning activity was studied by Falahah and Rosmala (2012). Social media was used by the teachers for task assignment, class announcements, scheduling and examination. The benefits of social media far outweigh the negative effects. The research suggested that use of social media for teaching learning activity is beneficial to both the users. Social media augments learning.

The current study aimed to gather information related to social media usage by the students with special reference to the type of information shared on social media; content watched; common social media platforms preferred by the subjects; advantages and disadvantages of using social network as perceived by the students.

\section{METHODOLOGY}

An exploratory research design was employed for the study. Based on purposive random sampling method, students actively using social media network were selected. A total of 200 students belonging to the government (Govt. College for women, Guntur, Govt. State University (ANU Guntur) and State Agriculture University (ANGRAU) participated in the study. A structured questionnaire was developed to gather information related to social media usage by the students, which was administered to the study group using a Google form in May 2020. Undergraduate students in the age group of 18-24 years were targeted due to their diverse nature. The specific research questions that guided the study were :

- Which among the social media was the most preferred by the students and what was the content shared on these sites?

- Is there any significant difference in the time spent on various social media?

- Is there a significant difference in the different content watched on social media?

- Can we compare the watched content to the shared content among different social media networks?
Data computation was done by using various statistical tests such as frequency, percentage, average, and standard deviation. Further, two- way anova test was used to find out the difference between the various social networks used and the content watched in these networks. Moreover, to know the significance difference between the content watched and shared through various social network sites F- test was computed. The null hypotheses developed for the study were tested at a 0.05 level of significance.

\section{RESULTS AND DISCUSSION}

It can be inferred from Table 1 that, a noteworthy segment of the respondents was sharing information by utilizing WhatsApp with an average of 77.16 followed by Instagram (29.16), YouTube (16.16), and Facebook (16.0). The reason for using WhatsApp is that it is very simple and easy to share information related to studies, announcements, etc., without the hassles of everyday login. A significant section of the respondents was sharing through Snapchat followed by Pinterest, Tiktok, and Google plus (15.00, 7.33, 7.00\& 6.16) correspondingly. A meager portion of the respondents was sharing information by using Twitter and Skype $(6.16 \& 4.33)$ respectively.

Aanu and Sisodia (2021) revealed that majority of the post graduation students belonging to three agriculture universities of Rajastha were using you tube followed by whatsapp and face book social networking sites to extend their learning opportunities and exchange information.

The most frequently used digital media to attain awareness on Swachh Bharath Mission was by using social media networking sites such as facebook, twitter, websites etc. (12.50\%), mobile applications (3.75\%), and IVR/SMS/Call campaigns (1.25\%) (Rohit et al., 2021)

Table 2 reveals that there is a significant difference between social media networks and the content shared on social media at 0.05 level of significance. From the ANOVA analysis, it is further revealed that among the social networks WhatsApp is the most sought-after one and it is used to share messages. The probable reason for this might be that messages are used for multiple purposes such as to provide, obtain and inform various matters related to academics, family and fun through WhatsApp considering its numerous advantages like personalization, the convenience of using local language, 
Table 1. College student's social media preference and type of information shared on social media

\begin{tabular}{lll}
\hline Type of social media & Av. & SD \\
\hline WhatsApp & 77.16 & 8.78 \\
Instagram & 29.16 & 5.40 \\
YouTube & 16.16 & 4.02 \\
Facebook & 16.00 & 4.00 \\
Snapchat & 15.00 & 3.87 \\
Pinterest & 7.33 & 2.70 \\
Tiktok & 7.00 & 2.64 \\
Google plus & 6.83 & 2.61 \\
Twitter & 6.16 & 2.48 \\
Skype & 4.33 & 2.08 \\
Most shared events in social media & & \\
Text Messages & 30.50 & 5.52 \\
Photos & 28.40 & 5.32 \\
Videos & 27.50 & 5.24 \\
Subject related information & 13.30 & 3.64 \\
Posting Personal events of daily life & 6.20 & 2.48 \\
Health tips & & \\
\hline
\end{tabular}

Table 2. Two-way ANOVA analysis to evaluate the most preferred social media network and the contents shared

\begin{tabular}{llll}
\hline Source of Variation & F- test & P-value & F-critical \\
\hline Social media networks & 10.82 & $1.01 \mathrm{E}-08$ & 2.09 \\
Contents shared on & 5.09 & 0.000877 & 2.42 \\
social media & & & \\
\hline
\end{tabular}

ability to track if the receiver has seen the message or not.

It is apparent from Table 3 that the majority (41.08) of the respondents were watching various content through YouTube followed by WhatsApp (18.25), Instagram (18.0), and Facebook (10.66). The reason for the high usage of YouTube may be due to the availability of all kinds of videos especially in the vernacular language where there is more possibility of connecting to the message content. WhatsApp being the most personalized app, it helps students to connect with friends, family, and professional groups. A considerable portion of the respondents was sharing content by using Tiktok, Pinterest, and Google plus with an average of $(6.25,6.00 \& 5.50)$ respectively. A limited number of respondents were using Snapchat, Twitter, and Skype with an average of $(4.08,3.08 \& 2.50)$. A cross-sectional study conducted in northern India reported that Facebook was found to be the most popular social media used by the study group (Saini et al. 2020). When it came to the type of content watched on
Table 3. The most common social media platforms used by the students to watch various content

\begin{tabular}{lll}
\hline Type of social media & Av. & SD \\
\hline Social Media & Av. & SD \\
YouTube & 41.08 & 14.53 \\
WhatsApp & 18.25 & 7.80 \\
Instagram & 18.00 & 6.70 \\
Facebook & 10.66 & 3.31 \\
Tiktok & 6.25 & 3.62 \\
Pinterest & 6.00 & 1.89 \\
Google plus & 5.50 & 2.19 \\
Snap chat & 4.08 & 3.18 \\
Twitter & 3.08 & 1.82 \\
Skype & 2.50 & 1.50 \\
Most watched events on social media & & \\
Music & 17.60 & 15.80 \\
Cooking & 12.60 & 16.63 \\
Movies & 11.15 & 14.93 \\
Health & 10.65 & 9.79 \\
Spiritual content & 8.80 & 8.37 \\
Embroidery/ painting & 8.45 & 8.26 \\
\hline
\end{tabular}

Table 4. Two-way ANOVA to evaluate the preferred social media network groups and content watched

\begin{tabular}{|c|c|c|}
\hline Source of Variation & $\mathrm{F}$ & P-value F critical \\
\hline Social Media Networks & 29.88 & 4.02E-16 2.09 \\
\hline Content watched on socia & 1 media & 0.0046292 .42 \\
\hline
\end{tabular}

different social media networks, most of the respondents were listening to music followed by cooking, movies with averages of $(17.6,12.6 \& 11.15)$ respectively. This could be attributed to the fact that most adolescents may connect and form into close-knit peer groups based on their interests and hobbies, especially related to music, food, and movies. The average per cent of the respondents were watching health tips (10.65), spiritual content (8.37), and embroidery \& painting (8.45) on social media, as these are highly personal preferences and very relevant for those who have expertise in these areas.

It can be read from Table 4 that there is a significant difference between watched social media networks and also there exists a significant difference between the different contents watched on the social media at 0.05 level of significance. The most watched content was music on YouTube. The reason for differences among the usage of social media networks and content may be connected to many factors such as expertise, security, safety, interests, and preferences. 
Table 5. Comparison of watched content and shared content among different social media networks

\begin{tabular}{llllll}
\hline Social Media & AVG & SD & F-test & P-value & F-critical \\
\hline Watched & 18.51 & 21.92 & 3.42 & 0.040 & 3.17 \\
Shared & 11.54 & 11.85 & & & \\
\hline
\end{tabular}

Table 6.Advantages and disadvantages of using social network

\begin{tabular}{lll}
\hline Advantages & No. & $\%$ \\
\hline Keeping in touch with friends is convenient & 178 & 89.00 \\
Learning technology becomes easier & 168 & 84.00 \\
To know about academic institutions for & 165 & 82.50 \\
higher studies across the country & & \\
To know the opinions of leaders, actors, & 153 & 76.50 \\
scientists, sportsmen & & \\
Accessing information around the globe & 151 & 75.50 \\
within minutes & & \\
Exchange of ideas and collaborations & 142 & 71.00 \\
Meeting new people can help you socialize & 123 & 61.50 \\
It is free & 113 & 56.50 \\
Disadvantages & & \\
Lesser physical activity & 152 & 76.00 \\
Cybercrime & 147 & 73.50 \\
Privacy issues & 142 & 71.00 \\
Addiction & 139 & 69.50 \\
Mental Illness & 138 & 69.00 \\
Misleading Information & 120 & 60.00 \\
Reduced focus on studies & 113 & 56.50 \\
Decreases social skills & 103 & 51.50 \\
\hline
\end{tabular}

There is a significant difference between watched and shared content in different social media networks. The likely reason for this might be that although a person may see messages daily, he/she only shares them if the message has impressed and motivated him/her highly. The need to share arises only if the person connects to the message on a personal level and intends to pass it on to friends and family (Table 5).

Hong Kong student's use of social media both in school and outside of school (Lu et.al., 2016). It was reported that content consuming and sharing activities were more than content creating activities. Both content consuming content sharing activities were significantly less frequent in school than outside of school. Interestingly, content creating activities were significantly more frequent in school than outside school.

The results from Table 6 indicate that a majority $(89 \%)$ of the respondents expressed that social network make it very easy for them to keep regularly in touch with their friends and family who are living far apart due to various reasons. Even for people living close by, social media has become the most sought after, instant way of updating friends and family with their latest successes and failures in life.

A highly significant (84\%) portion of the respondents articulated that it is easy to learn new technology features through the social network. The most likely reason for this might be the availability of ready-made interactive videos available in social networks, which makes learning much easier. A noteworthy \% of the respondents $(82.5 \%)$ believed that social networks are the most effective platforms to know about various academic institutions for higher studies across the country as they can get in touch with their seniors through these networks. Moreover, various academic institutions advertise about their institutions through social networks, project groups, and alumni groups active on social media which is an effective source of information for the students to know about the related institutes for higher education. (Balamurugan and Thanuskodi 2019) also reported that college students used social media for 'learning' (27\%) and 'sharing information' (22.6\%).

More than three fourth of the respondents stated that social media is one such platform to know the opinion of leaders, actors, scientists, and sportsmen depending upon their interest and that within minutes they can access information around the globe $(75.50 \%)$, and exchange ideas and collaborations (71\%). A significant per cent $(61.50 \%)$ of the respondents think that meeting new people through social media can help them to socialize. Another advantage stated was that social media network is free $(56.50 \%)$ i.e., if a person has internet they can access the sites and learn for free.

A majority of the students ( $76 \%$ ) believed that 'less physical activity' was the leading disadvantage of social media. The apparent reason for this might be that all of the respondents were undergraduates, a major portion of their time spent will be on academics, and after that their main form of recreation or relaxation is spending time on social media networks which, again is a sedentary task with no scope for physical activity. Cybercrime is another problem $(73.50 \%)$ faced by the respondents. This could be due to the ignorance of privacy settings and the inability to recognize the reliability of the sources of advertisements, friend requests, etc., and privacy issues (71\%). A study 
conducted in universities of north India revealed the problems faced by the students in using social media included wiling away of valuable time, fear of misuse of personal information, and lack of security and privacy (Singh and Gill, 2015). Survey among the college students of Tamil Nadu showed more than 'above average' level of awareness on cyber related threat issues. (Senthilkumar and Easwaramoorthy, 2017).

The respondents also reported that addiction (69.50\%), mental illness (69\%) and misleading information $(60 \%)$ are a few other problems faced. As the social network is an effective means to stay up-todate, and this is the status quo for this technology-reliant society. Moreover, getting likes to their shared posts motivate them to engage more time on a social network that may turn into an addiction and long-term usage. Consequently, spending more hours may result in a plethora of mental and physical problems.

More than $56.50 \%$ of the respondents professed that social media indulgence reduces their focus on studies and decreases social skills $(51.50 \%)$. As the students are spending their productive time on social media, their concentration on studies and contact with relatives, elders, and family members may be curtailed.

\section{CONCLUSION}

Analysis of college students' social media usage shows that WhatsApp is the most preferred medium employed to share text messages, photos, and videos. YouTube, WhatsApp, and Instagram were the top three platforms used to watch content related to music, cooking, and movies. Significant differences were observed between social media networks and content shared/watched and among the content watched and content shared on social media, demonstrating that the multitude of messages circulating daily has a varied and lasting impact on students. So students have considerable skills in accessing and using various social media skills which, can be utilized for enriching their academic experience and expertise.

\section{CONFLICTS OF INTEREST}

The authors declare that they have no conflicts of interest.

\section{REFERENCES}

Anil Kumar Malik and Godara, A.K. (2020). Information and communication technologies (ICTs) use by the students of CCSHAU, Hisar. Indian Res. J. Ext. Edu. 20 (4):14-19.

Annu, G. and Sisodia, S.S. (2021). Utilization pattern of social media among the postgraduate students. Indian Res. J. Ext. Edu. 21 (2\&3): 26-32.

Balamurugan. T. and Thanuskodi, S. (2019). Use of social networking sites among the college students in Tamil Nadu, India. Library Philosophy and Practice. 2301. Environment, Procedia-Social and Behavioral Sci., 67, 156-166.

Falahah, Rosmala, D. (2012). Study of Social Networking Usage in Higher Education Gratifications Theory Revisited. Intl. J.of Busi. and Social Sci., 4 (5) : 27-34.

Jha, V.K. (2017). A study on the engagement of Indian students on social media. J. of Content, Community \& Commu. 6(3): 50-55.

Lu, J.; Hao, Q. Jing, M. 2016. Consuming, sharing, and creating content: How young students use new social media in and outside school. Computers in Human Behavior, 64 (1): 55-64.

Rohit; Walake, C.; Singh, D.K.; Yadav, R.N. and Singh, L.B. (2021). Utilization pattern of parious sources of information for the awareness of "Swachh Bharat Mission" in Meerut district. Indian Res. J. Ext. Edu. 21(1) : 67-70

Saini, N; Sangwan, G..; Verma, M.; Kohli, A.; Kaur, M. and Lakshmi, P.V.M. (2020). Effect of social networking sites on the quality of life of college students: A cross-sectional study from a city in North India. The Scientific World J., 2020, 1-8.

Senthilkumar, K. and Easwaramoorthy, S. (2017). A survey on cyber security awareness among college students in Tamil Nadu. in IOP conference series: Materials Sci.and Engg., 263 (4) : 42-43. IOP Publishing.

Singh, K.P; Gill, M.S. (2015). Role and users' approach to social networking sites (SNSs): a study of universities of North India. The Electronic Library, 33 (1) : 19-34.

Tayseer, M.; Zoghieb, F.; Alcheikh, I. and Awadallah, M.N.S. (2014). Social network: Academic and social impact on college students. In: ASEE 2014 Zone I Conference, CT, USA. 